\title{
The n-Hosoya Polynomials of Some Classes of Thorn Graphs
}

Ali A. Ali Ahmed M. Ali

aliazizali1933@yahoo.com ahmedgraph@uomosul.edu.iq

College of Computer Sciences and Mathematics

University of Mosul

Received on:30/12/2008

Accepted on:17/3/2009

\section{ABSTRACT}

The n-Hosoya Polynomials of cog-complete graphs, thorn cogcomplete graphs , cog-stars, thorn cog-stars , cog-wheels , and thorn cogwheels are obtained. The n-Wiener indices of these graphs are also determined .

Keywords: cog-graph, thorn graph , n-distance , n-Hosoya polynomial ,nWiener index.

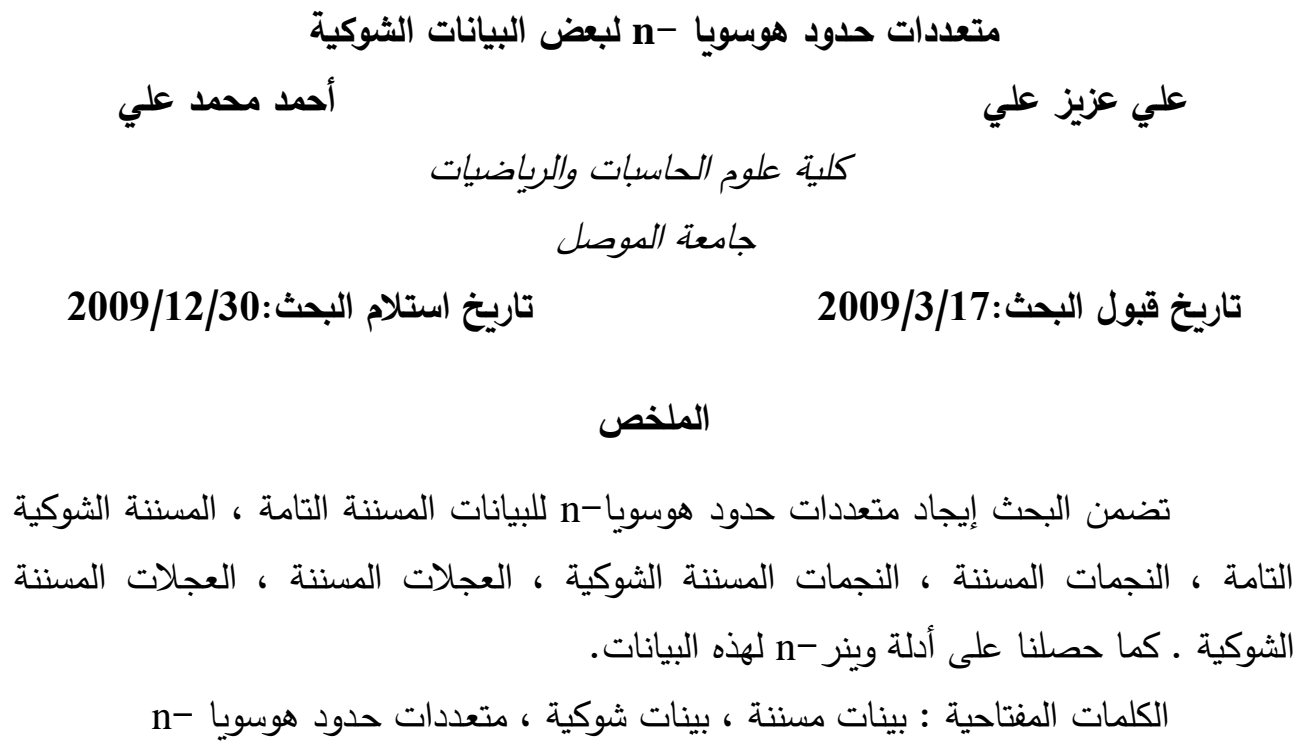

\section{الملخص}

تضمن البحث إيجاد متعددات حدود هوسويا-n للبيانات المسننة التامة ، المسننة الثوكية

التامة ، النجمات المسننة ، النجمات المسننة الثوكية ، العجلات المسننة ، العجلات المسننة

الشوكية . كما حصلنا على أدلة وينر -n لهذه البيانات.

n- الكلمات المفتاحية : بينات مسننة ، بينات شوكية ، متعددات حدود هوسويا

\section{Introduction:}

We follow the terminology of [3],[4]. Let $\mathrm{v}$ be a vertex of a connected graph $\mathrm{G}$, and let $\mathrm{S}$ be an (n-1)-subset of $\mathrm{V}(\mathrm{G}), n \geq 2$, then the n-distance $d_{n}(v, S)$ is defined by [1]

$$
d_{n}(v, S)=\min \{d(v, u): u \in S\} .
$$

The n-diameter of $\mathbf{G}$ is defined by

$$
\operatorname{diam}_{n} G=\max _{2}\left\{d_{n}(v, S): v \in V(G),|S|=n-1, S \subseteq V(G)\right\} .
$$

The $\mathbf{n}$-Wiener index of $\mathbf{G}$ is defined by 


$$
W_{n}(G)=\sum_{(v, S)} d_{n}(v, S) .
$$

The $\mathbf{n - H o s o y a}$ polynomial of connected graph $\mathbf{G}$ of order $\mathbf{p}$ is defined by

$$
H_{n}(G ; x)=\sum_{k=0}^{\delta_{n}} C_{n}(G, k) x^{k},
$$

where $3 \leq n \leq p, \delta_{n}$ is the n-diameter of $\mathrm{G}$, and $C_{n}(G, k)$ is the number of order pairs $(v, S), v \in V(G), S \subseteq V(G),|S|=n-1$, such that $d_{n}(v, S)=k$.

One can easily show that [1].

$C_{n}(G, 0)=p\left(\begin{array}{l}p-1 \\ n-2\end{array}\right), C_{n}(G, 1)=p\left(\begin{array}{l}p-1 \\ n-1\end{array}\right)-\sum_{v \in V(G)}\left(\begin{array}{c}p-1-\operatorname{deg} v \\ n-1\end{array}\right)$.

The n-Hosoya polynomial of a vertex $\mathbf{v}$ in $\mathbf{G}$, denoted by $H_{n}(v, G ; x)$, is defined [1] by

$$
H_{n}(v, G ; x)=\sum_{k \geq 0} C_{n}(v, G, k) x^{k},
$$

where $C_{n}(v, G, k)$ is the number of (n-1)-subsets of vertices $\mathrm{S}$ such that $d_{n}(v, S)=k$. It is clear that for each $\mathrm{k}, 0 \leq k \leq \delta_{n}$,

$$
C_{n}(G, k)=\sum_{v \in V(G)} C_{n}(v, G, k),
$$

and

$$
H_{n}(G ; x)=\sum_{v \in V(G)} H_{n}(v, G ; x),
$$

The following simple lemma is useful for obtaining $C_{n}(v, G, k)$ for every vertex $\mathrm{v}$ of a connected graph $\mathrm{G}$.

Lemma 1.1: [2] Let $t$ be the number of vertices of ordinary distance $k$ from vertex $\mathrm{v}$, and let $\mathrm{s}$ be the number of vertices of distance more than $\mathrm{k}$ from $\mathrm{v}$ in a connected graph $\mathrm{G}$. Then

$$
C_{n}(v, G, k)=\left(\begin{array}{l}
s+t \\
n-1
\end{array}\right)-\left(\begin{array}{c}
s \\
n-1
\end{array}\right),
$$

for $v \in V(G), 2 \leq n \leq p, 0 \leq k \leq \delta_{n}$.

Let $\mathrm{T}$ be a non-empty subset of vertices of $\mathrm{G}$. We define

$$
C_{n}(T, G, k)=\sum_{v \in T} C_{n}(v, G, k) .
$$

We shall use this notation in our proofs. 
In this paper, we obtain n-Hosoya polynomials and n-Wiener indices of some classes of graphs that are not considered in the published research papers up to date as far as we know.

\section{Thorn Cog-Complete Graphs:}

The n-Hosoya polynomial will be obtained first for a cog-complete graph that is defined as follows.

Definition 2.1: A cog-complete graph $K_{m}^{c}$ is the graph constructed from a complete graph $K_{m}, m \geq 3$, of vertex set $\left\{v_{1}, v_{2}, \ldots, v_{m}\right\}$ with m additional vertices $u_{1}, u_{2}, \ldots, u_{m}$, and $2 \mathrm{~m}$ edges $\left\{u_{i} v_{i}, u_{i} v_{i+1}: i=1,2, \ldots, m\right\},\left(v_{m+1} \equiv v_{1}\right)$, as shown in Fig. 2.1.

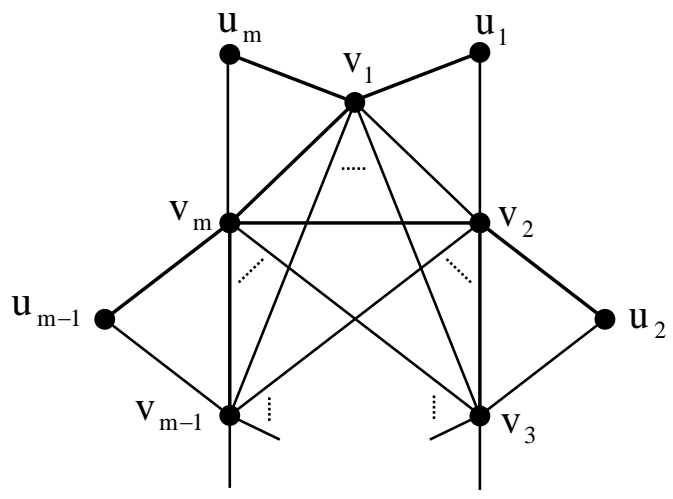

Fig. 2.1. $K_{m}^{c}$

It is clear that $p\left(K_{m}^{c}\right)=2 m, q\left(K_{m}^{c}\right)=\frac{1}{2} m(m+3)$, and for $m \geq 4$, diam $K_{m}^{c}=3$.

Moreover

$\operatorname{diam}_{n} K_{m}^{c}=\left\{\begin{array}{l}3, \text { if } 2 \leq n \leq m-2, m \geq 4 \\ 2, \text { if } m-1 \leq n \leq 2 m-2, \\ 1, \text { if } 2 m-1 \leq n \leq 2 m .\end{array}\right.$

Proposition 2.2: For $m \geq 3,3 \leq n \leq 2 m$, we have

$H_{n}\left(K_{m}^{c} ; x\right)=2 m\left(\begin{array}{c}2 m-1 \\ n-2\end{array}\right)+\sum_{k=1}^{3} C_{n}\left(K_{m}^{c}, k\right) x^{k}$,

where

$$
\begin{aligned}
& C_{n}\left(K_{m}^{c}, 1\right)=2 m\left(\begin{array}{c}
2 m-1 \\
n-1
\end{array}\right)-m\left[\left(\begin{array}{c}
m-2 \\
n-1
\end{array}\right)+\left(\begin{array}{c}
2 m-3 \\
n-1
\end{array}\right)\right], \\
& C_{n}\left(K_{m}^{c}, 2\right)=m\left[\left(\begin{array}{c}
2 m-3 \\
n-1
\end{array}\right)+\left(\begin{array}{c}
m-3 \\
n-2
\end{array}\right)\right],
\end{aligned}
$$


$C_{n}\left(K_{m}^{c}, 3\right)=m\left(\begin{array}{c}m-3 \\ n-1\end{array}\right)$.

Proof: The coefficients $C_{n}\left(K_{m}^{c}, 0\right)$ and $C_{n}\left(K_{m}^{c}, 1\right)$ are obtained from (1.5).

The coefficient $C_{n}\left(K_{m}^{c}, 3\right)$ is obtained by taking $v=u_{i}, 1 \leq i \leq m$, and the (n-1)subset $\mathrm{S}$ from the $\mathrm{m}-3$ vertices $u_{j}, j \neq i-1, i, i+1$. Then , $C_{n}\left(K_{m}^{c}, 2\right)$ is obtained from the fact $\sum_{k \geq 1} C_{n}(G, k)=p\left(\begin{array}{l}p-1 \\ n-1\end{array}\right)$.

The n-Wiener index of $K_{m}^{c}$ is given by

$W_{n}\left(K_{m}^{c}\right)=m\left[2\left(\begin{array}{c}2 m-1 \\ n-1\end{array}\right)+\left(\begin{array}{c}2 m-3 \\ n-1\end{array}\right)+\left(\begin{array}{c}m-2 \\ n-1\end{array}\right)+\left(\begin{array}{c}m-3 \\ n-1\end{array}\right)\right]$.

From Proposition 2.2 , we get the next corollary.

Corollary2.3: The Hosoya polynomial and the Wiener index of $K_{m}^{c}, m \geq 3$, are given by:

$H\left(K_{m}^{c} ; x\right)=2 m+\frac{1}{2} m(m+3) x+m(m-1) x^{2}+\frac{1}{2} m(m-3) x^{3}$.

And

$W\left(K_{m}^{c}\right)=m(4 m-5)$.

Now, we define a thorn cog- complete graph and find its n-Hosoya polynomial.

Definition 2.4: A thorn cog-complete graph , denoted by $K_{m}^{c^{*}}$, is the cogcomplete graph $K_{m}^{c}, m \geq 3$ constructed in Definition 2.1, with $2 \mathrm{~m}$ additional endvertices $w_{1}, w_{2}, \ldots, w_{2 m}$, and edges $\left\{u_{i} w_{2 i-1}, u_{i} w_{2 i}: i=1,2, \ldots, m\right\}$, as shown in Fig. 2.2.

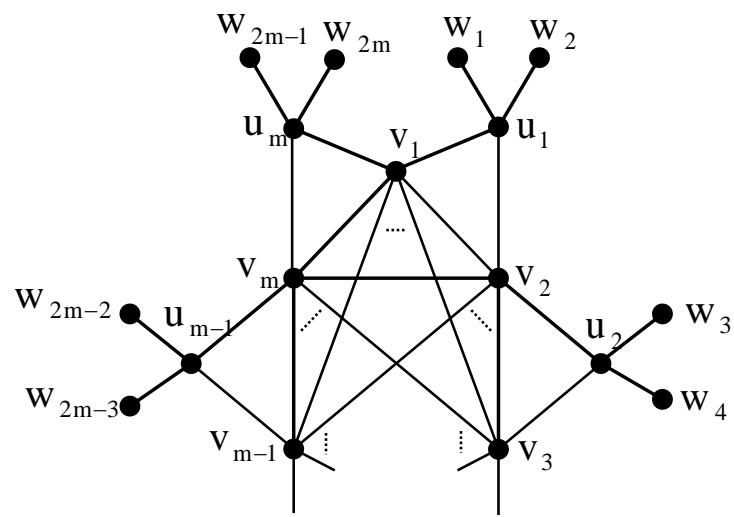

Fig.2.2. $K_{m}^{c^{*}}$ 
It is clear that $p\left(K_{m}^{c^{*}}\right)=4 m, q\left(K_{m}^{c^{*}}\right)=\frac{1}{2} m(m+7)$, and, diam $K_{m}^{c^{*}}=5$, for $m \geq 4$.

The $\mathrm{n}$-diameter is as given below:

$$
\operatorname{diam}_{n} K_{m}^{c^{*}}=\left\{\begin{array}{l}
5, \text { if } 2 \leq n \leq 2 m-5, m \geq 4 \\
4, \text { if } 2 m-4 \leq n \leq 3 m-4, \\
3, \text { if } 3 m-3 \leq n \leq 4 m-4, \\
2, \text { if } 4 m-3 \leq n \leq 4 m-1, \\
1, \text { if } n=4 m .
\end{array}\right.
$$

The n-Hosoya polynomial of $K_{m}^{c^{*}}$ is given in the next theorem.

Theorem 2.4: The n-Hosoya polynomial of $K_{m}^{c^{*}}, m \geq 3,3 \leq n \leq 4 m$ is given by $H_{n}\left(K_{m}^{c^{*}} ; x\right)=4 m\left(\begin{array}{c}4 m-1 \\ n-2\end{array}\right)+\sum_{k=1}^{5} C_{n}\left(K_{m}^{c^{*}}, k\right) x^{k}$,

where

$$
\begin{aligned}
& C_{n}\left(K_{m}^{c^{*}}, 1\right)=4 m\left(\begin{array}{c}
4 m-1 \\
n-1
\end{array}\right)-m\left[\left(\begin{array}{c}
3 m-2 \\
n-1
\end{array}\right)+\left(\begin{array}{c}
4 m-5 \\
n-1
\end{array}\right)+2\left(\begin{array}{c}
4 m-2 \\
n-1
\end{array}\right)\right], \\
& C_{n}\left(K_{m}^{c^{*}}, 2\right)=m\left[\left(\begin{array}{c}
3 m-2 \\
n-1
\end{array}\right)-\left(\begin{array}{c}
3 m-5 \\
n-1
\end{array}\right)-\left(\begin{array}{c}
4 m-5 \\
n-1
\end{array}\right)-\left(\begin{array}{c}
2 m-4 \\
n-1
\end{array}\right)\right]+2 m\left(\begin{array}{c}
4 m-2 \\
n-1
\end{array}\right), \\
& C_{n}\left(K_{m}^{c^{*}}, 3\right)=m\left[2\left(\begin{array}{c}
4 m-5 \\
n-1
\end{array}\right)+\left(\begin{array}{c}
2 m-4 \\
n-1
\end{array}\right)-\left(\begin{array}{c}
3 m-5 \\
n-1
\end{array}\right)-\left(\begin{array}{c}
2 m-6 \\
n-1
\end{array}\right)\right], \\
& C_{n}\left(K_{m}^{c^{*}}, 4\right)=m\left[2\left(\begin{array}{c}
3 m-5 \\
n-1
\end{array}\right)-\left(\begin{array}{c}
2 m-6 \\
n-1
\end{array}\right)\right], \\
& C_{n}\left(K_{m}^{c^{*}}, 5\right)=2 m\left(\begin{array}{c}
2 m-6 \\
n-1
\end{array}\right) .
\end{aligned}
$$

Proof: Using (1.5) we get $C_{n}\left(K_{m}^{c^{*}}, 1\right)$ as given in (2.2).

We partition the vertex set of $K_{m}^{c^{*}}$ into three subsets $\mathrm{W}, \mathrm{U}$, and $\mathrm{V}$, where $W=\left\{w_{1}, w_{2}, \ldots, w_{2 m}\right\}, U=\left\{u_{1}, u_{2}, \ldots, u_{m}\right\}$, and $V=\left\{v_{1}, v_{2}, \ldots, v_{m}\right\}$.

From Fig 2.2., for $\mathrm{k}=2,3,4,5$, one can easily notice that:

and

$$
\begin{aligned}
& C_{n}\left(W, K_{m}^{c^{*}}, k\right)=2 m C_{n}\left(w_{i}, K_{m}^{c^{*}}, k\right), \text { for any vertex } w_{i} \in W, \\
& C_{n}\left(U, K_{m}^{c^{*}}, k\right)=m C_{n}\left(u_{i}, K_{m}^{c^{*}}, k\right), \text { for any vertex } u_{i} \in U,
\end{aligned}
$$

$$
C_{n}\left(V, K_{m}^{c^{*}}, k\right)=m C_{n}\left(v_{i}, K_{m}^{c^{*}}, k\right), \text { for any vertex } v_{i} \in V .
$$

Thus, we have three cases for the values of $\mathrm{k}$.

Case(1): $\mathrm{k}=3$. There are $\mathrm{m}$ vertices, namely $u_{2} ; v_{3}, v_{4}, \ldots, v_{m-1}, v_{m} ; u_{m}$, of distance 3 from $w_{l}$; and there are (3m-5) vertices of distance more than 3 from $\mathrm{w}_{1}$. Therefore, by Lemma 1.1 , 


$$
C_{n}\left(w_{1}, K_{m}^{c^{*}}, 3\right)=\left(\begin{array}{c}
4 m-5 \\
n-1
\end{array}\right)-\left(\begin{array}{c}
3 m-5 \\
n-1
\end{array}\right) .
$$

Also , there are $(\mathrm{m}+1)$ vertices , namely $w_{3}, w_{4} ; u_{3}, u_{4}, \ldots, u_{m-2}, u_{m-1} ; w_{2 m-1}$ , $w_{2 m}$, of distance 3 from $\mathrm{u}_{1}$; and there are $(2 \mathrm{~m}-6)$ vertices of distance more than 3 from $u_{l}$. Therefore

$$
C_{n}\left(u_{1}, K_{m}^{c^{*}}, 3\right)=\left(\begin{array}{c}
3 m-5 \\
n-1
\end{array}\right)-\left(\begin{array}{c}
2 m-6 \\
n-1
\end{array}\right) .
$$

Finally, there are (2m-4) vertices , namely $w_{3}, w_{4}, \ldots, w_{2 m-3}, w_{2 m-2}$, of distance 3 from $v_{1}$; and there is no vertex of distance more than 3 from $v_{1}$. Hence

$$
C_{n}\left(v_{1}, K_{m}^{c^{*}}, 3\right)=\left(\begin{array}{c}
2 m-4 \\
n-1
\end{array}\right) .
$$

Thus from (2.7), (2.8), and (2.9) we get (2.4).

Case(2): $\mathrm{k}=4$. There are $(\mathrm{m}+1)$ vertices , namely $w_{3}, w_{4} ; u_{3}, u_{4}, \ldots, u_{m-2}$, $u_{m-1} ; w_{2 m-1}, w_{2 m}$, of distance 4 from $\mathrm{w}_{1}$; and there are $(2 \mathrm{~m}-6)$ vertices of distance more than 4 from $w_{1}$. Therefore, by Lemma 1.1 ,

$$
C_{n}\left(w_{1}, K_{m}^{c^{*}}, 4\right)=\left(\begin{array}{c}
3 m-5 \\
n-1
\end{array}\right)-\left(\begin{array}{c}
2 m-6 \\
n-1
\end{array}\right) .
$$

Also , there are (2m-6) vertices, namely $w_{5}, w_{6}, \ldots, w_{2 m-3}, w_{2 m-2}$, of distance 4 from $u_{l}$; and no vertex of distance more than 4 from $u_{l}$. Therefore

$$
C_{n}\left(u_{1}, K_{m}^{c^{*}}, 4\right)=\left(\begin{array}{c}
2 m-6 \\
n-1
\end{array}\right) .
$$

Finally, there is no vertex of distance more than 4 from any vertex of V. Thus, from (2.10) and (2.11) we obtain (2.5).

Case(3): $\mathrm{k}=5$. There are $(2 \mathrm{~m}-6)$ vertices , namely $w_{5}, w_{6}, \ldots, w_{2 m-3}, w_{2 m-2}$, of distance 5 from $w_{1}$; and no vertex of distance more than 5 from $w_{1}$.

Also, there is no vertex of distance 5 from any vertex of $V \cup U$. Thus we get (2.6).

From (2.2), (2.4), (2.5), (2.6) and using (2.1) we get (2.3).

Hence, the proof is completed

Corollary 2.5: The $\mathrm{n}$-Wiener index of $K_{m}^{c^{*}}, \mathrm{~m} \geq 3,3 \leq \mathrm{n} \leq 4 \mathrm{~m}$ is given by $W_{n}\left(K_{m}^{c^{*}}\right)=4 m\left(\begin{array}{c}4 m-1 \\ n-1\end{array}\right)+3 m\left[\left(\begin{array}{c}4 m-5 \\ n-1\end{array}\right)+\left(\begin{array}{c}3 m-5 \\ n-1\end{array}\right)+\left(\begin{array}{c}2 m-6 \\ n-1\end{array}\right)\right]$ 


$$
+m\left[2\left(\begin{array}{c}
4 m-2 \\
n-1
\end{array}\right)+\left(\begin{array}{c}
3 m-2 \\
n-1
\end{array}\right)+\left(\begin{array}{c}
2 m-4 \\
n-1
\end{array}\right)\right] .
$$

Corollary 2.6: The Hosoya polynomial of $K_{m}^{c^{*}}, \mathrm{~m} \geq 3$ is given by

$$
\begin{aligned}
H\left(K_{m}^{c^{*}} ; x\right) & =4 m+\frac{1}{2} m(m+7) x+m(m+4) x^{2}+\frac{1}{2} m(5 m-3) x^{3} \\
& +2 \mathrm{~m}(\mathrm{~m}-1) \mathrm{x}^{4}+2 \mathrm{~m}(\mathrm{~m}-3) \mathrm{x}^{5} .
\end{aligned}
$$

Proof: When $\mathrm{n}=2$, we have $d_{2}\left(u^{\prime},\left\{v^{\prime}\right\}\right)=d_{2}\left(v^{\prime},\left\{u^{\prime}\right\}\right)=d\left(u^{\prime}, v^{\prime}\right)$.

Thus $H\left(K_{m}^{c^{*}} ; x\right)$ is obtained from Theorem 2.4 , by putting $\mathrm{n}=2$ and dividing by 2 .

Corollary 2.7: The Wiener index of $K_{m}^{c^{*}}, m \geq 3$ is given by $W\left(K_{m}^{c^{*}}\right)=m(28 m-31)$.

\section{Thorn Cog-star Graphs:}

Definition 3.1: A cog-star graph $S_{m}^{c}$ is the graph constructed from a star [4] $, S_{m}, m \geq 4$, of vertex set $\left\{v_{1}, v_{2}, \ldots, v_{m-1}, v_{m}\right\}$ with (m-1) additional vertices $u_{1}, u_{2}, \ldots, u_{m-2}, u_{m-1}$, and edges $\left\{u_{i} v_{i+1}, u_{i} v_{i+2}: i=1,2, \ldots, m-1\right\}$, $\left(v_{m+l} \equiv v_{2}\right)$, as shown in Fig. 3.1.

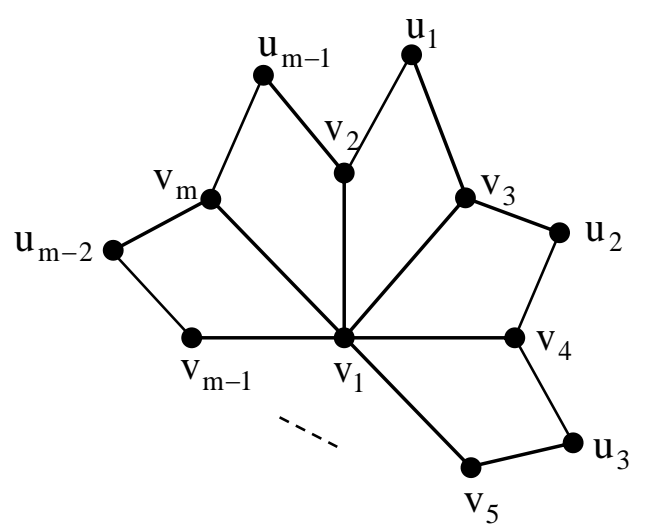

Fig. 3.1. $S_{m}^{c}$

It is clear that $p\left(S_{m}^{c}\right)=2 m-1, q\left(S_{m}^{c}\right)=3(m-1)$, diam $S_{m}^{c}=4$ for $m \geq 5$, and $\operatorname{diam}_{n} S_{m}^{c} \leq 4$.

From Fig.3.1. , we notice that there are (m-3) vertices of distance 3 from $u_{i}$; and there are (m-4) vertices of distance more than 3 from $u_{i}$, for $i=1,2, \ldots, m-1$. Thus by Lemma 1.1 ,

$$
C_{n}\left(u_{i}, S_{m}^{c}, 3\right)=\left(\begin{array}{c}
2 m-7 \\
n-1
\end{array}\right)-\left(\begin{array}{l}
m-4 \\
n-1
\end{array}\right), i=1,2, \ldots, m-1 .
$$


Also , there are (m-3) vertices of distance 3 from $v_{i}$; and no vertex of distance more than 3 from $v_{i}, i=2,3, \ldots, m$, thus

$$
C_{n}\left(v_{i}, S_{m}^{c}, 3\right)=\left(\begin{array}{l}
m-3 \\
n-1
\end{array}\right), i=2,3, \ldots, m .
$$

Thus, from (3.1), and (3.2) we get

$$
C_{n}\left(S_{m}^{c}, 3\right)=(m-1)\left[\left(\begin{array}{c}
2 m-7 \\
n-1
\end{array}\right)+\left(\begin{array}{c}
m-4 \\
n-2
\end{array}\right)\right] .
$$

Moreover

$$
C_{n}\left(S_{m}^{c}, 4\right)=(m-1)\left(\begin{array}{c}
m-4 \\
n-1
\end{array}\right) .
$$

Using (1.5), we get

$$
C_{n}\left(S_{m}^{c}, 1\right)=(2 m-1)\left(\begin{array}{c}
2 m-2 \\
n-1
\end{array}\right)-(m-1)\left[\left(\begin{array}{c}
2 m-4 \\
n-1
\end{array}\right)+\left(\begin{array}{c}
2 m-5 \\
n-1
\end{array}\right)\right]-\left(\begin{array}{c}
m-1 \\
n-1
\end{array}\right) .
$$

Thus , from (1.10), (3.3), (3.4), and (3.5), we obtain

$$
C_{n}\left(S_{m}^{c}, 2\right)=(m-1)\left[\left(\begin{array}{c}
2 m-4 \\
n-1
\end{array}\right)+\left(\begin{array}{c}
2 m-5 \\
n-1
\end{array}\right)-\left(\begin{array}{c}
2 m-7 \\
n-1
\end{array}\right)-\left(\begin{array}{c}
m-3 \\
n-1
\end{array}\right)\right]+\left(\begin{array}{c}
m-1 \\
n-1
\end{array}\right) \ldots
$$

Hence, we have the following theorem :

Theorem 3.1: The n-Hosoya polynomial of $S_{m}^{c}, m \geq 4,3 \leq n \leq 2 m-1$ is given by

$$
H_{n}\left(S_{m}^{c} ; x\right)=(2 m-1)\left(\begin{array}{c}
2 m-2 \\
n-2
\end{array}\right)+\sum_{k=1}^{4} C_{n}\left(S_{m}^{c}, k\right) x^{k},
$$

where $C_{n}\left(S_{m}^{c}, k\right), \mathrm{k}=1,2,3,4$ are given in (3.5), (3.6), (3.3), and (3.4), respectively.

Corollary 3.2: The $\mathrm{n}$-Wiener index of $S_{m}^{c}, m \geq 4,3 \leq n \leq 2 m-1$ is given by

$$
\begin{aligned}
W_{n}\left(S_{m}^{c}\right) & =(m-1)\left[\left(\begin{array}{c}
2 m-7 \\
n-1
\end{array}\right)+\left(\begin{array}{c}
2 m-5 \\
n-1
\end{array}\right)+\left(\begin{array}{c}
2 m-4 \\
n-1
\end{array}\right)+\left(\begin{array}{c}
m-3 \\
n-1
\end{array}\right)+\left(\begin{array}{c}
m-4 \\
n-1
\end{array}\right)\right] . \\
& +(2 m-1)\left(\begin{array}{c}
2 m-2 \\
n-1
\end{array}\right)+\left(\begin{array}{c}
m-1 \\
n-1
\end{array}\right) .
\end{aligned}
$$

Definition 3.3: The thorn cog-star $S_{m}^{c^{*}}$ is the graph constructed from the $\operatorname{cog}$-star $S_{m}^{c}, \mathrm{~m} \geq 4$, of vertex set $\left\{\mathrm{v}_{1}, \mathrm{v}_{2}, \ldots, \mathrm{v}_{\mathrm{m}}, \mathrm{u}_{1}, \mathrm{u}_{2}, \ldots, \mathrm{u}_{\mathrm{m}-1}\right\}$ (Fig.3.1) with $2(\mathrm{~m}-1)$ additional vertices $\mathrm{w}_{1}, \mathrm{w}_{2}, \ldots, \mathrm{w}_{2 \mathrm{~m}-3}, \mathrm{w}_{2 \mathrm{~m}-2}$, and edges $\left\{\mathrm{u}_{\mathrm{i}} \mathrm{w}_{2 \mathrm{i}-1}, \mathrm{u}_{\mathrm{i}} \mathrm{w}_{2 \mathrm{i}}: \mathrm{i}=1,2, \ldots, \mathrm{m}-1\right\}$, as shown in Fig. 3.2. 


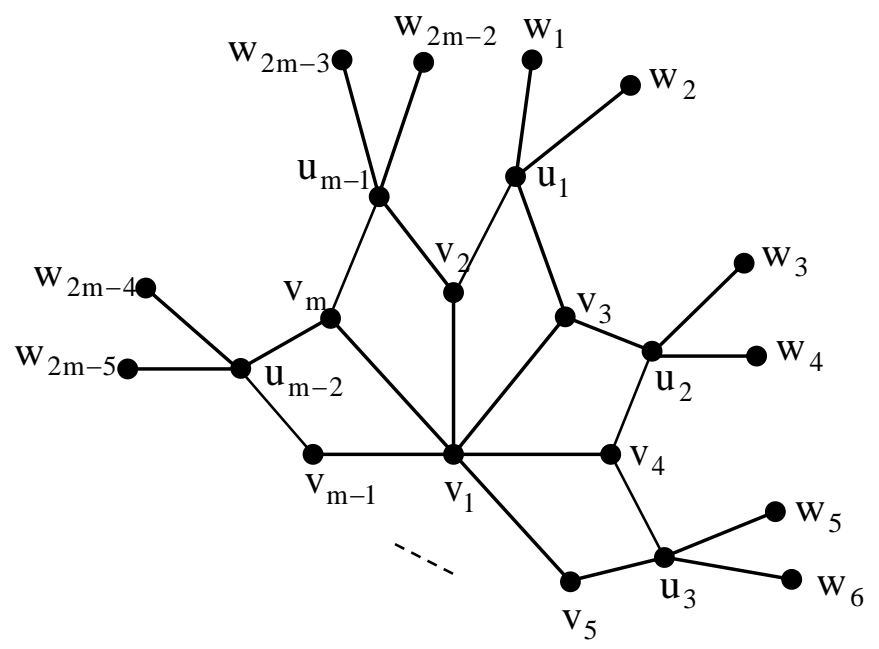

Fig. 3.2. $S_{m}^{c^{*}}$

It is clear that $p\left(S_{m}^{c^{*}}\right)=4 m-3, q\left(S_{m}^{c^{*}}\right)=5(m-1)$, diam $S_{m}^{c^{*}}=6$ for $m \geq 5$, and $\operatorname{diam}_{n} S_{m}^{c^{*}} \leq 6$.

In the next theorem, we obtain the n-Hosoya polynomial of $S_{m}^{c^{*}}, m \geq 4$.

Theorem 3.4: The n-Hosoya polynomial of $S_{m}^{c^{*}}, m \geq 4,3 \leq n \leq 4 m-3$ is given by

$H_{n}\left(S_{m}^{c^{*}} ; x\right)=(4 m-3)\left(\begin{array}{c}4 m-4 \\ n-2\end{array}\right)+\sum_{k=1}^{6} C_{n}\left(S_{m}^{c^{*}}, k\right) x^{k}$,

where

$$
\begin{aligned}
C_{n}\left(S_{m}^{c^{*}}, 1\right)= & (4 m-3)\left(\begin{array}{c}
4 m-4 \\
n-1
\end{array}\right)-(m-1)\left[2\left(\begin{array}{c}
4 m-5 \\
n-1
\end{array}\right)+\left(\begin{array}{c}
4 m-7 \\
n-1
\end{array}\right)+\left(\begin{array}{c}
4 m-8 \\
n-1
\end{array}\right)\right]-\left(\begin{array}{c}
3 m-3 \\
n-1
\end{array}\right), \\
C_{n}\left(S_{m}^{c^{*}}, 2\right) & =2(m-1)\left(\begin{array}{c}
4 m-5 \\
n-1
\end{array}\right)-(m-1)\left[\left(\begin{array}{c}
3 m-9 \\
n-1
\end{array}\right)+\left(\begin{array}{c}
4 m-11 \\
n-1
\end{array}\right)-\left(\begin{array}{c}
4 m-8 \\
n-2
\end{array}\right)\right] \\
& +\left(\begin{array}{c}
3 m-3 \\
n-1
\end{array}\right)-\left(\begin{array}{c}
2 m-2 \\
n-1
\end{array}\right), \\
C_{n}\left(S_{m}^{c^{*}}, 3\right)= & (m-1)\left[\left(\begin{array}{c}
3 m-9 \\
n-1
\end{array}\right)-\left(\begin{array}{c}
4 m-11 \\
n-1
\end{array}\right)-\left(\begin{array}{c}
3 m-12 \\
n-1
\end{array}\right)-\left(\begin{array}{c}
2 m-6 \\
n-1
\end{array}\right)\right]+\left(\begin{array}{c}
2 m-2 \\
n-1
\end{array}\right) \\
& +2(m-1)\left(\begin{array}{c}
4 m-8 \\
n-1
\end{array}\right)
\end{aligned}
$$




$$
\begin{aligned}
& C_{n}\left(S_{m}^{c^{*}}, 4\right)=(m-1)\left[\left(\begin{array}{c}
2 m-6 \\
n-1
\end{array}\right)-\left(\begin{array}{c}
2 m-8 \\
n-1
\end{array}\right)-\left(\begin{array}{c}
3 m-12 \\
n-1
\end{array}\right)\right]+2(m-1)\left(\begin{array}{c}
4 m-11 \\
n-1
\end{array}\right), \\
& C_{n}\left(S_{m}^{c^{*}}, 5\right)=(m-1)\left[2\left(\begin{array}{c}
3 m-12 \\
n-1
\end{array}\right)-\left(\begin{array}{c}
2 m-8 \\
n-1
\end{array}\right)\right]
\end{aligned}
$$

and

$C_{n}\left(S_{m}^{c^{*}}, 6\right)=2(m-1)\left(\begin{array}{c}2 m-8 \\ n-1\end{array}\right)$.

Proof: $C_{n}\left(S_{m}^{c^{*}}, 1\right)$ follows from (1.5). Now we find $C_{n}\left(S_{m}^{c^{*}}, k\right)$, for $\mathrm{k}=3,4,5,6$. From Fig.3.2, we notice that

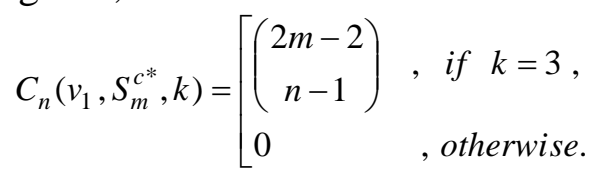

Let $V=\left\{v_{2}, v_{3}, \ldots, v_{m}\right\}, U=\left\{u_{1}, u_{2}, \ldots, u_{m-1}\right\}$, and $W=\left\{w_{1}, w_{2}, \ldots, w_{2 m-2}\right\}$. There are (m-3) vertices of distance 3 from any vertex $v \in V$; for example ; each of $u_{2}, u_{3}, \ldots, u_{m-2}$, is of distance 3 from $\mathrm{v}_{2}$; and there are (2m-6) vertices of distance more than 3 from $v_{2}$. Hence, by Lemma 1.1,

$$
C_{n}\left(v_{i}, S_{m}^{c^{*}}, 3\right)=\left(\begin{array}{c}
3 m-9 \\
n-1
\end{array}\right)-\left(\begin{array}{c}
2 m-6 \\
n-1
\end{array}\right), 2 \leq i \leq m .
$$

Moreover, there are $(m+1)$ vertices of distance 3 from any vertex $\mathrm{u} \in \mathrm{U}$; for example, each of $\mathrm{w}_{3}, \mathrm{w}_{4} ; \mathrm{v}_{4}, \mathrm{v}_{5}, \ldots, \mathrm{v}_{\mathrm{m}} ; \mathrm{w}_{2 \mathrm{~m}-3}, \mathrm{w}_{2 \mathrm{~m}-2}$ is of distance 3 from $\mathrm{u}_{1}$; and there are $(3 \mathrm{~m}-12)$ vertices of distance more than 3 from $\mathrm{u}_{1}$. Hence, by Lemma 1.1 ,

$$
C_{n}\left(u_{i}, S_{m}^{c^{*}}, 3\right)=\left(\begin{array}{c}
4 m-11 \\
n-1
\end{array}\right)-\left(\begin{array}{c}
3 m-12 \\
n-1
\end{array}\right), 1 \leq i \leq m-1 .
$$

Finally, there are three vertices of distance 3 from any vertex $\mathrm{w} \in \mathrm{W}$; and there are (4m-11) vertices of distance more than 3 from $w$. Hence, by Lemma 1.1,

$$
C_{n}\left(w_{i}, S_{m}^{c^{*}}, 3\right)=\left(\begin{array}{c}
4 m-8 \\
n-1
\end{array}\right)-\left(\begin{array}{c}
4 m-11 \\
n-1
\end{array}\right), 1 \leq i \leq 2 m-2 .
$$

From (3.14) - (3.17), we get (3.10).

When $\mathrm{k}=4$, there are (2m-6) vertices, of distance 4 from any vertex $\mathrm{v} \in \mathrm{V}$; and there is no vertex of graph $S_{m}^{c^{*}}$ of distance more than 4 from v. Hence

$$
C_{n}\left(v_{i}, S_{m}^{c^{*}}, 4\right)=\left(\begin{array}{c}
2 m-6 \\
n-1
\end{array}\right), 2 \leq i \leq m .
$$

And there are (m-4) vertices of distance 4 from any vertex $u \in U$; for example , each of $u_{3}, u_{4}, u_{5}, \ldots, u_{m-3}, u_{m-2}$ is of distance 4 from $u_{1}$; and 
there are $(2 \mathrm{~m}-8)$ vertices of distance more than 4 from $u_{1}$. Hence, by Lemma 1.1 ,

$$
C_{n}\left(u_{i}, S_{m}^{c^{*}}, 4\right)=\left(\begin{array}{c}
3 m-12 \\
n-1
\end{array}\right)-\left(\begin{array}{c}
2 m-8 \\
n-1
\end{array}\right), 1 \leq i \leq m-1 .
$$

Moreover, there are $(\mathrm{m}+1)$ vertices of distance 4 from any vertex $w \in W$; for example, each of $w_{3}, w_{4} ; v_{4}, v_{5}, \ldots, v_{m} ; w_{2 m-3}, w_{2 m-2}$ is of distance 4 from $w_{1}$; and there are (3m-12) vertices of distance more than 4 from $w_{1}$. Hence, by Lemma 1.1,

$$
C_{n}\left(w_{i}, S_{m}^{c^{*}}, 4\right)=\left(\begin{array}{c}
4 m-11 \\
n-1
\end{array}\right)-\left(\begin{array}{c}
3 m-12 \\
n-1
\end{array}\right), 1 \leq i \leq 2 m-2 .
$$

Thus, from (3.18), (3.19), and (3.20), we get (3.11).

Now, when $\mathrm{k}=5$, then $C_{n}\left(v_{i}, S_{m}^{c^{*}}, 5\right)=0,2 \leq i \leq m$

Moreover, there are (2m-8) vertices of distance 5 from any vertex $u \in U$; and there is no vertex of graph $S_{m}^{c^{*}}$ of distance more than 5 from $\mathrm{u}$. Hence

$$
C_{n}\left(u_{i}, S_{m}^{c^{*}}, 5\right)=\left(\begin{array}{c}
2 m-8 \\
n-1
\end{array}\right), 1 \leq i \leq m-1 \text {. }
$$

And there are $(\mathrm{m}-4)$ vertices of distance 5 from any vertex $\mathrm{w} \in \mathrm{W}$; for example, each of the vertices $u_{3}, u_{4}, u_{5}, \ldots, u_{m-3}, u_{m-2}$ is of distance 5 from $\mathrm{w}_{1}$; and there are (2m-8) vertices of distance more than 5 from $w_{1}$. Hence, by Lemma 1.1 ,

$$
C_{n}\left(w_{i}, S_{m}^{c^{*}}, 5\right)=\left(\begin{array}{c}
3 m-12 \\
n-1
\end{array}\right)-\left(\begin{array}{c}
2 m-8 \\
n-1
\end{array}\right), 1 \leq i \leq 2 m-2 .
$$

Thus, from (3.21), and (3.22), we get (3.12).

Finally, from Fig.3.2, we notice that

$$
\begin{aligned}
& C_{n}\left(v_{i}, S_{m}^{c^{*}}, 6\right)=0,2 \leq i \leq m, \\
& C_{n}\left(u_{i}, S_{m}^{c^{*}}, 6\right)=0,1 \leq i \leq m-1,
\end{aligned}
$$

and

$$
C_{n}\left(w_{i}, S_{m}^{c^{*}}, 6\right)=\left(\begin{array}{c}
2 m-8 \\
n-1
\end{array}\right), 1 \leq i \leq 2 m-2 .
$$

Using (2.1) we obtain , from (3.8) and (3.10) - (3.13), the value of $C_{n}\left(S_{m}^{c^{*}}, 2\right)$ as given in (3.9).

This completes the proof .

Corollary 3.5: The $\mathrm{n}$-Wiener index of $S_{m}^{c^{*}}, m \geq 4,3 \leq n \leq 4 m-3$ is given by

$$
\begin{aligned}
W_{n}\left(S_{m}^{c^{*}}\right)= & (4 m-3)\left(\begin{array}{c}
4 m-4 \\
n-1
\end{array}\right)+2(m-1)\left(\begin{array}{c}
4 m-5 \\
n-1
\end{array}\right)+\left(\begin{array}{c}
3 m-3 \\
n-1
\end{array}\right)+\left(\begin{array}{c}
2 m-2 \\
n-1
\end{array}\right) \\
& +3(m-1)\left[\left(\begin{array}{c}
2 m-8 \\
n-1
\end{array}\right)+\left(\begin{array}{c}
4 m-8 \\
n-1
\end{array}\right)+\left(\begin{array}{c}
4 m-11 \\
n-1
\end{array}\right)+\left(\begin{array}{c}
3 m-12 \\
n-1
\end{array}\right)\right]
\end{aligned}
$$




$$
+(m-1)\left[\left(\begin{array}{c}
4 m-7 \\
n-1
\end{array}\right)+\left(\begin{array}{c}
3 m-9 \\
n-1
\end{array}\right)+\left(\begin{array}{c}
2 m-6 \\
n-1
\end{array}\right)\right] .
$$

Corollary 3.6: If $S_{m}^{c^{*}}$ is the thorn cog-star of order $4 \mathrm{~m}-3$, then the Hosoya polynomial of $S_{m}^{c^{*}}, m \geq 4$ is given by

$$
\begin{aligned}
H\left(S_{m}^{c^{*}} ; x\right)= & 4 m-3+5(m-1) x+\frac{1}{2}(m-1)(m+12) x^{2}+(m-1)(m+3) x^{3} \\
& +\frac{1}{2}(m-1)(5 m-8) x^{4}+2(m-1)(m-4) x^{5}+2(m-1)(m-4) x^{6} .
\end{aligned}
$$

And, the Wiener index of $S_{m}^{c^{*}}, m \geq 4$, is given by $W\left(S_{m}^{c^{*}}\right)=36 m^{2}-114 m+78$.

\section{Thorn Cog-wheel Graphs:}

Definition 4.1: A cog-wheel graph $W_{m}^{c}$ is the graph constructed from a wheel [4] $, W_{m}, m \geq 4$, of order $\mathrm{m}$, with vertex set $\left\{v_{1}, v_{2}, \ldots, v_{m-1}, v_{m}\right\}$, and with (m-1) additional vertices $u_{1}, u_{2}, \ldots, u_{m-2}, u_{m-1}$, and edges $\left\{u_{i} v_{i+1}, u_{i} v_{i+2}: i=1,2, \ldots, m-1\right\},\left(v_{m+1} \equiv v_{2}\right)$, as shown in Fig. 4.1.

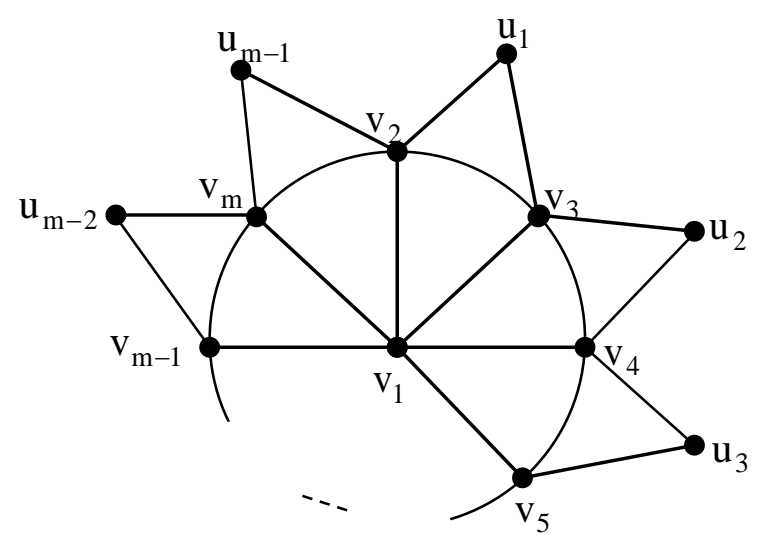

Fig. 4.1. $W_{m}^{c}$

It is clear that $p\left(W_{m}^{c}\right)=2 m-1, q\left(W_{m}^{c}\right)=4(m-1), \operatorname{diam} W_{m}^{c}=4$ for $m \geq 7$, and $\operatorname{diam}_{n} W_{m}^{c} \leq 4$.

From Fig.4.1., we notice that :

$$
\begin{aligned}
C_{n}\left(u_{i}, W_{m}^{c}, 3\right) & =\sum_{j=1}^{n-1}\left(\begin{array}{c}
m-3 \\
j
\end{array}\right)\left(\begin{array}{c}
m-6 \\
n-j-1
\end{array}\right) \\
& =\left(\begin{array}{c}
2 m-9 \\
n-1
\end{array}\right)-\left(\begin{array}{c}
m-6 \\
n-1
\end{array}\right), i=1,2, \ldots, m-1, m \geq 6,
\end{aligned}
$$




$$
C_{n}\left(v_{i}, W_{m}^{c}, 3\right)=\left(\begin{array}{c}
m-5 \\
n-1
\end{array}\right), i=2,3, \ldots, m, m \geq 5,
$$

Thus, from (4.1), and (4.2) we get

$$
C_{n}\left(W_{m}^{c}, 3\right)=(m-1)\left[\left(\begin{array}{c}
2 m-9 \\
n-1
\end{array}\right)+\left(\begin{array}{c}
m-6 \\
n-2
\end{array}\right)\right], m \geq 6 .
$$

Moreover

$$
C_{n}\left(W_{m}^{c}, 4\right)=(m-1)\left(\begin{array}{c}
m-6 \\
n-1
\end{array}\right), m \geq 6 .
$$

Using (1.5), we obtain

$$
C_{n}\left(W_{m}^{c}, 1\right)=(2 m-1)\left(\begin{array}{c}
2 m-2 \\
n-1
\end{array}\right)-(m-1)\left[\left(\begin{array}{c}
2 m-4 \\
n-1
\end{array}\right)+\left(\begin{array}{c}
2 m-7 \\
n-1
\end{array}\right)\right]-\left(\begin{array}{c}
m-1 \\
n-1
\end{array}\right) .
$$

Finally , from $(4.3),(4.4),(4.5)$, and (2.1), we get

$$
C_{n}\left(W_{m}^{c}, 2\right)=(m-1)\left[\left(\begin{array}{c}
2 m-4 \\
n-1
\end{array}\right)+\left(\begin{array}{c}
2 m-7 \\
n-1
\end{array}\right)-\left(\begin{array}{c}
2 m-9 \\
n-1
\end{array}\right)-\left(\begin{array}{c}
m-5 \\
n-1
\end{array}\right)\right]+\left(\begin{array}{c}
m-1 \\
n-1
\end{array}\right) .
$$

Hence, we have the following results :

Theorem 4.1: The n-Hosoya polynomial of $W_{m}^{c}, m \geq 6,3 \leq n \leq 2 m-1$ is given by $H_{n}\left(W_{m}^{c} ; x\right)=(2 m-1)\left(\begin{array}{c}2 m-2 \\ n-2\end{array}\right)+\sum_{k=1}^{4} C_{n}\left(W_{m}^{c}, k\right) x^{k}$,

where $C_{n}\left(W_{m}^{c}, k\right), \mathrm{k}=1,2,3,4$ are given in (4.5), (4.6), (4.3), and (4.4), respectively.

Corollary 4.2: The $\mathrm{n}$-Wiener index of $W_{m}^{c}, m \geq 6,3 \leq n \leq 2 m-1$ is given by

$$
\begin{aligned}
W_{n}\left(W_{m}^{c}\right)= & (m-1)\left[\left(\begin{array}{c}
2 m-4 \\
n-1
\end{array}\right)+\left(\begin{array}{c}
2 m-7 \\
n-1
\end{array}\right)+\left(\begin{array}{c}
2 m-9 \\
n-1
\end{array}\right)+\left(\begin{array}{c}
m-5 \\
n-1
\end{array}\right)+\left(\begin{array}{c}
m-6 \\
n-1
\end{array}\right)\right] \\
& +(2 m-1)\left(\begin{array}{c}
2 m-2 \\
n-1
\end{array}\right)+\left(\begin{array}{c}
m-1 \\
n-1
\end{array}\right) .
\end{aligned}
$$

\section{Remarks (4.1):}

(i) For $3 \leq n \leq 7$, we have

$$
\text { - } H_{n}\left(W_{4}^{c} ; x\right)=7\left(\begin{array}{c}
6 \\
n-2
\end{array}\right)+\left[7\left(\begin{array}{c}
6 \\
n-1
\end{array}\right)-3\left(\begin{array}{c}
4 \\
n-1
\end{array}\right)-\left(\begin{array}{c}
3 \\
n-1
\end{array}\right)\right] x+\left[3\left(\begin{array}{c}
4 \\
n-1
\end{array}\right)+\left(\begin{array}{c}
3 \\
n-1
\end{array}\right)\right] x^{2} \text {. }
$$

(ii) For $3 \leq n \leq 9$, we have

- $H_{n}\left(W_{5}^{c} ; x\right)=9\left(\begin{array}{c}8 \\ n-2\end{array}\right)+\left[9\left(\begin{array}{c}8 \\ n-1\end{array}\right)-4\left(\begin{array}{c}6 \\ n-1\end{array}\right)-4\left(\begin{array}{c}3 \\ n-1\end{array}\right)-\left(\begin{array}{c}4 \\ n-1\end{array}\right)\right] x$ 


$$
+\left[4\left(\begin{array}{c}
6 \\
n-1
\end{array}\right)+4\left(\begin{array}{c}
3 \\
n-1
\end{array}\right)+\left(\begin{array}{c}
4 \\
n-1
\end{array}\right)\right] x^{2} \cdot \#
$$

Definition 4.3: The thorn cog-wheel graph $W_{m}^{c^{*}}$ is the graph constructed from the cog- wheel graph $W_{m}^{c}, m \geq 4$, of vertex set $\left\{v_{1}, v_{2}, \ldots, v_{m}, u_{1}, u_{2}, \ldots, u_{m-1}\right\}$ (Fig.4.1) with 2(m-1) additional vertices $w_{1}, w_{2}, \ldots, w_{2 m-3}, w_{2 m-2}$, and edges $\left\{u_{i} w_{2 i-1}, u_{i} w_{2 i}: i=1,2, \ldots, m-1\right\}$, as shown in Fig. 4.2.

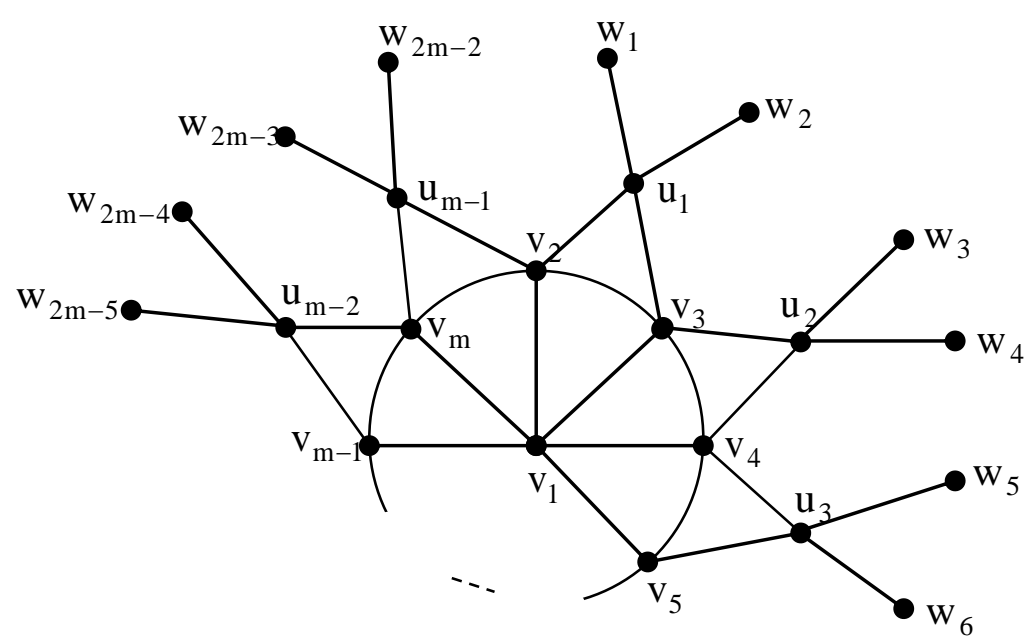

Fig. 4.2. $W_{m}^{c^{*}}$

It is clear that $p\left(W_{m}^{c^{*}}\right)=4 m-3, q\left(W_{m}^{c^{*}}\right)=6(m-1)$, diam $W_{m}^{c^{*}}=6$ for $m \geq 7$, and $\operatorname{diam}_{n} W_{m}^{c^{*}} \leq 6$.

Using the procedure followed for obtaining $H_{n}\left(S_{m}^{c^{*}} ; x\right)$, we establish the next theorem, which determines $H_{n}\left(W_{m}^{c^{*}} ; x\right)$.

Theorem 4.4: If $W_{m}^{c^{*}}$ is the thorn cog-wheel of order $4 \mathrm{~m}-3, m \geq 6$, $3 \leq n \leq 4 m-3$, then

$H_{n}\left(W_{m}^{c^{*}} ; x\right)=(4 m-3)\left(\begin{array}{c}4 m-4 \\ n-2\end{array}\right)+\sum_{k=1}^{6} C_{n}\left(W_{m}^{c^{*}}, k\right) x^{k}$,

where

$C_{n}\left(W_{m}^{c^{*}}, 1\right)=(4 m-3)\left(\begin{array}{c}4 m-4 \\ n-1\end{array}\right)-(m-1)\left[2\left(\begin{array}{c}4 m-5 \\ n-1\end{array}\right)+\left(\begin{array}{c}4 m-8 \\ n-1\end{array}\right)+\left(\begin{array}{c}4 m-9 \\ n-1\end{array}\right)\right]-\left(\begin{array}{c}3 m-3 \\ n-1\end{array}\right)$, 


$$
\begin{aligned}
C_{n}\left(W_{m}^{c^{*}}, 2\right) & =(m-1)\left[2\left(\begin{array}{c}
4 m-5 \\
n-1
\end{array}\right)+\left(\begin{array}{c}
4 m-9 \\
n-1
\end{array}\right)-\left(\begin{array}{c}
4 m-8 \\
n-2
\end{array}\right)-\left(\begin{array}{c}
4 m-13 \\
n-1
\end{array}\right)-\left(\begin{array}{c}
3 m-11 \\
n-1
\end{array}\right)\right] \\
& +\left(\begin{array}{c}
3 m-3 \\
n-1
\end{array}\right)-\left(\begin{array}{c}
2 m-2 \\
n-1
\end{array}\right), \\
C_{n}\left(W_{m}^{c^{*}}, 3\right) & =(m-1)\left[2\left(\begin{array}{c}
4 m-8 \\
n-1
\end{array}\right)+\left(\begin{array}{c}
3 m-11 \\
n-1
\end{array}\right)-\left(\begin{array}{c}
4 m-13 \\
n-1
\end{array}\right)-\left(\begin{array}{c}
3 m-14 \\
n-1
\end{array}\right)-\left(\begin{array}{c}
2 m-10 \\
n-1
\end{array}\right)\right] \\
& +\left(\begin{array}{c}
2 m-2 \\
n-1
\end{array}\right), \\
C_{n}\left(W_{m}^{c^{*}}, 4\right) & =(m-1)\left[2\left(\begin{array}{c}
4 m-13 \\
n-1
\end{array}\right)+\left(\begin{array}{c}
2 m-10 \\
n-1
\end{array}\right)-\left(\begin{array}{c}
3 m-14 \\
n-1
\end{array}\right)-\left(\begin{array}{c}
2 m-12 \\
n-1
\end{array}\right)\right], \\
C_{n}\left(W_{m}^{c^{*}}, 5\right) & =(m-1)\left[2\left(\begin{array}{c}
3 m-14 \\
n-1
\end{array}\right)-\left(\begin{array}{c}
2 m-12 \\
n-1
\end{array}\right)\right],
\end{aligned}
$$

and

$$
C_{n}\left(W_{m}^{c^{*}}, 6\right)=2(m-1)\left(\begin{array}{c}
2 m-12 \\
n-1
\end{array}\right) \text {. }
$$

\section{Remarks (4.2):}

(i) For $3 \leq n \leq 13$, we have

- $H_{n}\left(W_{4}^{c^{*}} ; x\right)=13\left(\begin{array}{c}12 \\ n-2\end{array}\right)+\left[13\left(\begin{array}{c}12 \\ n-1\end{array}\right)-6\left(\begin{array}{c}11 \\ n-1\end{array}\right)-3\left(\begin{array}{c}8 \\ n-1\end{array}\right)-3\left(\begin{array}{c}7 \\ n-1\end{array}\right)-\left(\begin{array}{c}9 \\ n-1\end{array}\right)\right] x$

$$
\begin{aligned}
& +\left[6\left(\begin{array}{c}
11 \\
n-1
\end{array}\right)+\left(\begin{array}{c}
9 \\
n-1
\end{array}\right)-\left(\begin{array}{c}
6 \\
n-1
\end{array}\right)+3\left[\left(\begin{array}{c}
7 \\
n-1
\end{array}\right)-\left(\begin{array}{c}
8 \\
n-1
\end{array}\right)-\left(\begin{array}{c}
4 \\
n-1
\end{array}\right)-\left(\begin{array}{c}
2 \\
n-1
\end{array}\right)\right]\right] x^{2} \\
& +\left[6\left(\begin{array}{c}
8 \\
n-1
\end{array}\right)+3\left(\begin{array}{c}
2 \\
n-1
\end{array}\right)+\left(\begin{array}{c}
6 \\
n-1
\end{array}\right)-3\left(\begin{array}{c}
4 \\
n-1
\end{array}\right)\right] x^{3}+6\left(\begin{array}{c}
4 \\
n-1
\end{array}\right) x^{4} .
\end{aligned}
$$

(ii) For $3 \leq n \leq 17$, we have

$$
\begin{aligned}
-H_{n}\left(W_{5}^{c^{*} ;} ;\right. & =17\left(\begin{array}{c}
16 \\
n-2
\end{array}\right)+\left[17\left(\begin{array}{c}
16 \\
n-1
\end{array}\right)-8\left(\begin{array}{c}
15 \\
n-1
\end{array}\right)-5\left(\begin{array}{c}
12 \\
n-1
\end{array}\right)-4\left(\begin{array}{c}
11 \\
n-1
\end{array}\right)\right] x \\
+ & {\left[8\left(\begin{array}{c}
15 \\
n-1
\end{array}\right)+4\left(\begin{array}{c}
11 \\
n-1
\end{array}\right)-3\left(\begin{array}{c}
12 \\
n-1
\end{array}\right)-4\left(\begin{array}{c}
7 \\
n-1
\end{array}\right)-4\left(\begin{array}{c}
4 \\
n-1
\end{array}\right)-\left(\begin{array}{c}
8 \\
n-1
\end{array}\right)\right] x^{2} } \\
+ & {\left[4\left(2\left(\begin{array}{c}
12 \\
n-1
\end{array}\right)+\left(\begin{array}{c}
4 \\
n-1
\end{array}\right)-\left(\begin{array}{c}
7 \\
n-1
\end{array}\right)-\left(\begin{array}{c}
2 \\
n-1
\end{array}\right)\right]+\left(\begin{array}{c}
8 \\
n-1
\end{array}\right)\right] x^{3} } \\
+ & {\left[8\left(\begin{array}{c}
7 \\
n-1
\end{array}\right)-4\left(\begin{array}{c}
2 \\
n-1
\end{array}\right)\right] x^{4}+8\left(\begin{array}{c}
2 \\
n-1
\end{array}\right) x^{5} . \# }
\end{aligned}
$$

Corollary 4.5: The $\mathrm{n}$-Wiener index of $W_{m}^{c^{*}}$, of order $4 m-3 \quad m \geq 6$, $3 \leq n \leq 4 m-3$ is given by 


$$
\begin{aligned}
W_{n}\left(W_{m}^{c^{*}}\right) & =(4 m-3)\left(\begin{array}{c}
4 m-4 \\
n-1
\end{array}\right)+\left(\begin{array}{c}
3 m-3 \\
n-1
\end{array}\right)+\left(\begin{array}{c}
2 m-2 \\
n-1
\end{array}\right)+(m-1)\left[\left(\begin{array}{c}
4 m-9 \\
n-1
\end{array}\right)+\left(\begin{array}{c}
3 m-11 \\
n-1
\end{array}\right)\right. \\
& \left.+\left(\begin{array}{c}
2 m-10 \\
n-1
\end{array}\right)+2\left(\begin{array}{c}
4 m-5 \\
n-1
\end{array}\right)+3\left(\begin{array}{c}
4 m-8 \\
n-1
\end{array}\right)+3\left(\begin{array}{c}
4 m-13 \\
n-1
\end{array}\right)+3\left(\begin{array}{c}
3 m-14 \\
n-1
\end{array}\right)+3\left(\begin{array}{c}
2 m-12 \\
n-1
\end{array}\right)\right]
\end{aligned}
$$

Corollary 4.6: If $W_{m}^{c^{*}}$ is the thorn cog-wheel of order $4 m-3, m \geq 6$, then the Hosoya polynomial of $W_{m}^{c^{*}}$ is given by

$$
\begin{aligned}
H\left(W_{m}^{c^{*}} ; x\right) & =4 m-3+6(m-1) x+\frac{1}{2}(m-1)(m+14) x^{2}+(m-1)(m+6) x^{3} \\
+ & \frac{5}{2}(m-1)(m-2) x^{4}+2(m-1)(m-4) x^{5}+2(m-1)(m-6) x^{6} .
\end{aligned}
$$

Moreover

$H\left(W_{4}^{c^{*}} ; x\right)=13+18 x+24 x^{2}+24 x^{3}+12 x^{4}$,

and

$H\left(W_{5}^{c^{*}} ; x\right)=17+24 x+38 x^{2}+42 x^{3}+24 x^{4}+8 x^{5}$.

\section{Corollary 4.7:}

The Wiener index of $W_{m}^{c^{*}}$ of order $4 \mathrm{~m}-3, m \geq 6$, is given by $W\left(W_{m}^{c^{*}}\right)=2(m-1)(18 m-47)$,

Moreover

$W\left(W_{4}^{c^{*}}\right)=186$, and $W\left(W_{5}^{c^{*}}\right)=362$. 


\section{REFERENCES}

[1] Ali , A.A. , and Ali , A.M. ; (2006). "Wiener Polynomials of the Generalized Distance for some special Graphs", Raf. J. Comp. Sc. And Maths. Vol.3 , No.2 , pp.103-120.

[2] Ali , A.A., and Ahmed , H.G. ; (2007). "n-Wiener Polynomials of the m-Cube, Wagner Graphs and Thorn Stars", J. Dohuk University, Vol.10, No.2,

[3] Buckley , F. and Harary , F.; (1990). Distance in Graphs. Addison -Wesley, Redwood, California .

[4] Chartrand G. and Lesniak , L.; (1986). Graphs and Digraphs, $2^{\text {nd }}$, , Wadsworth and Brooks / Cole, California .

[5] Saeed, W.A.M. ; (1999) . “Wiener Polynomials of Graphs “, Ph.D. thesis, Mosul University, Mosul. 